\title{
Microinjection molding of polyamide 6/carbon nanotube composites
}

\author{
T. Ferreira ${ }^{1}$, P. E. Lopes ${ }^{2}$, M. C. Paiva*1 and A. J. Pontes ${ }^{1}$ \\ ${ }^{1}$ IPC/i3N - Institute for Polymers and Composites, University of Minho, Campus de Azurém, 4800-058 Guimarães, \\ Portugal \\ ${ }^{2}$ Pole for Innovation in Polymer Engineering - PIEP, University of Minho, 4800-058 Guimarães, Portugal
}

\begin{abstract}
Microinjection molding of polymer composites with carbon nanotubes (CNT) requires previous production of the nanocomposites, often by melt extrusion. Each processing step has a thermo-mechanical effect on the polymer melt, conveying different properties to the final product. In this work, polyamide 6 and its composites with pristine and functionalized CNT ( $\mathrm{f}-\mathrm{CNT}$ ) were processed by a mini twin-screw extrusion, followed

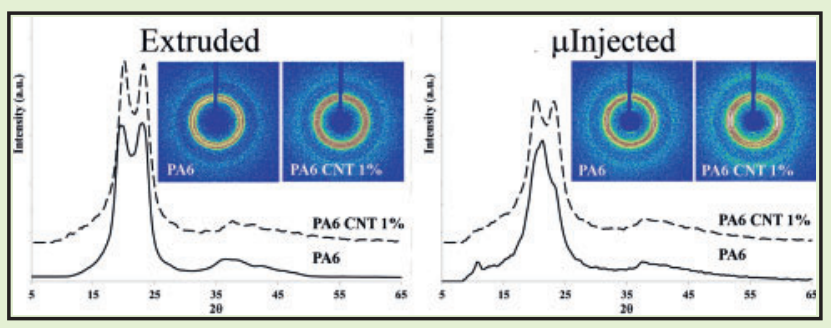
by microinjection molding. The morphology induced on the polymer by each process was analyzed by differential scanning calorimetry and wide angle X-ray diffraction. Calorimetric analysis showed a secondary crystallization for the microinjected materials, absent for the extruded materials. The characterization of microinjected polyamide 6 by $X$-ray diffraction revealed a large contribution of the $\gamma$ phase to the total crystallinity, mainly in the skin region, while the nanocomposites and extruded materials were characterized by a larger contribution of the $\alpha$ phase. Functionalization of CNT did not affect significantly the polymer morphology compared to composites with pristine CNT.
\end{abstract}

Keywords Nanocomposite, Polymer-matrix composites (PMCs), Physical properties, X-ray characterization, Injection molding, Extrusion

Cite this article T. Ferreira, P. E. Lopes, M. C. Paiva and A. J. Pontes. Nanocomposites, 2015, 1, 145-151

\section{Introduction}

Composites with polymers and carbon nanotubes (CNT) have been attracting the attention of research and industrial communities. The excellent electrical/thermal conductivity and high mechanical properties of CNT coupled with their nanoscale size and low density classify them as ideal onedimensional polymer reinforcement to generate nanocomposites with a wide range of potential applications. ${ }^{1-4}$

With the increasing demand for small and even microscale parts for CNT/polymer composites, microinjection molding $(\mu \mathrm{IM})$ became particularly desirable considering its largescale production ability and low production cost.

During $\mu \mathrm{MM}$, the polymer melt is subject to a complex thermo-mechanical history due to high injection pressure, high shear, high cooling rate, and very short cycle time that may affect the properties of the microparts $(\mu \mathrm{P}) .^{5-7}$ Besides these harsh processing conditions, the properties of the matrix may change due to the presence of the CNT that exhibit very large surface area, and may disturb the polymer flow, molecular orientation, and crystallization.

*Corresponding author, email mcpaiva@ dep.uminho.pt
Polyamide 6 (PA6), a semicrystalline polymer, exhibits high mechanical performance, good processability, and chemical resistance and is widely used in industry. Its physical and mechanical properties depend considerably on the crystalline structure and on the degree of crystallinity. ${ }^{8}$ The PA6 displays polymorphism, as it may crystallize in two different phases, the $\alpha$ - and $\gamma$-phase. In general, $\gamma$-phase is formed during fast cooling and low temperature crystallization, while slow cooling promotes the formation of the $\alpha$-phase., ${ }^{9,10}$

Few studies are reported in the literature concerning the crystallization behavior of PA reinforced with CNT. ${ }^{11-13}$ These nanocomposites seem to present different characteristics from those incorporating clays, the latter favoring the formation of the $\gamma$-phase. ${ }^{14,15}$ It is reported that the CNT may act as nucleation agents, favoring the formation of the $\alpha$ phase of PA6, inducing an increase in the crystallization degree, ${ }^{11-13}$ and the development of a second crystallization peak in nanocomposites observed by differential scanning calorimetry (DSC) studies. ${ }^{10,12,16}$ Coleman et al. report that a crystalline layer forms around the nanotubes that, combined with good interfacial adhesion, enhances the mechanical properties. ${ }^{17}$ However, other works report that high CNT concentrations, mainly with functionalized CNT (f-CNT) with a stronger compatibility with PA6, ${ }^{18,19}$ hinder the mobility of 
PA6 chains, delay the crystal growth and thus lead to the decrease of crystallinity relative to neat PA6. ${ }^{20-23}$

Limited literature is found concerning the crystalline characteristics of $\mu \mathrm{P}$ of neat polymers ${ }^{24-27}$ and polymer/CNT nanocomposites..$^{22,23}$ Only recently, Abassi et al ${ }^{28}$ reported the effect of the processing method on the crystallinity of nanocomposites and concluded that compression, $\mu \mathrm{IM}$, and $\mu \mathrm{IM}$ compression do not dramatically influence the crystallinity.

The present work focuses the study of extruded and $\mu \mathrm{IM}$ PA6/CNT nanocomposites with pristine and f-CNT. The production of $\mu \mathrm{IM}$ composite parts typically requires two processing steps, the first for compounding, usually by extrusion, and then the $\mu \mathrm{IM}$. These two melt processing methods are characterized by quite different process parameters, in particular the level of shear stress applied to the melt, its temperature, and cooling rate. These process dissimilarities may induce different states of nanoparticle dispersion and different polymer morphology, considerably affecting the final composite properties. Previous work by the authors reported the detailed study of the dispersion of CNT in extruded and $\mu$ IM PA6 composites. ${ }^{29}$ The production conditions of these nanocomposites as well as the nanocomposites mechanical and electrical characterization was reported there. Considerable differences in CNT dispersion were observed after extrusion and $\mu \mathrm{IM}$, while CNT functionalization displayed a smaller influence. ${ }^{29}$ These effects were observed to affect the mechanical and electrical properties of the resulting composites. The question remains about the magnitude of the influence of the processing method on the PA6 nanocomposites morphology developed. Wide angle X-ray diffraction analysis (WAXD) and DSC studies performed on extruded and $\mu \mathrm{IM}$ PA6 showed orientation effects at the skin of $\mu \mathrm{IM}$ parts as well as a predominance of the $\gamma$ crystalline form, and lower overall crystallinity compared to extruded PA6. ${ }^{27}$ The present work reports a detailed study of the PA6 morphology developed in the nanocomposites with low and high CNT and f-CNT content (1 and 4.5wt-\%, respectively), after extrusion and after $\mu \mathrm{IM}$ investigated by DSC and WAXD.

\section{Experimental methods}

\section{Materials and sample preparation}

Polyamide 6 used in this study was Badamid ${ }^{\circledR}$ LA70 K. The CNT used were NC7000 from Nanocyl, and were functionalized by 1,3-dipolar cycloaddition reaction, as reported elsewhere. ${ }^{30}$ The reaction was carried out under solvent-free conditions, inducing the thermal decomposition of paraformaldehyde into formaldehyde in the presence of $\mathrm{N}$-benzyloxycarbonylglycine at $250^{\circ} \mathrm{C}$ for $3 \mathrm{~h}$. The 1,3-dipole formed may react with the CNT surface, yielding pyrrolidine-f-CNT. ${ }^{30}$ The PA6/CNT composites containing 1 and $4.5 \mathrm{wt}-\%$ of asreceived CNT ( $p-C N T$ ) and $f-C N T$, designated by P1, F1, P4.5, and $\mathrm{F} 4.5$, respectively, were prepared via melt-mixing using a mini twin-screw extruder prototype. The neat PA6 was also extruded under similar conditions. The extruded rod was cooled in water at room temperature and then pelletized. Finally, the pelletized and dried extrudate was $\mu \mathrm{IM}$ as described in previous work ${ }^{29}$ to form millimeter-scale 'dog-bone' shaped bars with $300 \mu \mathrm{m}$ thickness, using a Boy 12 microinjection molding machine.

\section{Composite characterization Morphology}

Samples of extruded and $\mu \mathrm{IM}$ PA6/p-CNT and PA6/f-CNT nanocomposites were cryogenically fractured under liquid nitrogen. The cryo-fractured surfaces were sputtered with a thin layer of palladium-gold and observed by scanning electron microscopy (SEM) on a FEI Quanta 400 FEG ESEM.

\section{Differential scanning calorimetry}

Differential scanning calorimetry analysis was performed under nitrogen flow in the temperature range of $30-250^{\circ} \mathrm{C}$ on a Perkin Elmer Diamond Pyris equipment. The $\mu \mathrm{IM}$ samples were cut in the central region of the tensile specimen. Samples were heated to $250^{\circ} \mathrm{C}$ and held at that temperature for $2 \mathrm{~min}$ to remove any previous thermal history and then cooled from the melt to $30^{\circ} \mathrm{C}$. The scanning rates for heating and cooling during DSC measurements were set at $10^{\circ} \mathrm{Cmin}^{-1}$. For PA6/CNT nanocomposites, the equation used to calculate the polymer degree of crystallinity $\left(\chi_{c}\right)$ was corrected to account for the CNT weight fraction, as represented in equation (1)

$$
\chi_{c}(\%)=\frac{\Delta H_{f}}{(1-\Phi) \Delta H_{f}^{0}} \times 100
$$

Where $\phi$ is the weight fraction of CNTs in the composite, $\Delta H_{\mathrm{f}}$ is the heat of fusion of the analyzed sample (Joule per gram), and a $\Delta H_{\mathrm{f}}^{0}$ value of $188 \mathrm{~J} \mathrm{~g}^{-1}$ was used for PA6. ${ }^{31}$

\section{Wide angle $\mathrm{X}$-ray diffraction}

X-ray diffraction patterns were recorded using a CCD Bruker APEX II using graphite monochromatized Mo $\mathrm{K}_{\alpha}$ radiation $(\lambda=0.0711 \mathrm{~nm})$. Microinjection molding specimens of nanocomposite samples were mounted vertically and analyzed across the overall thickness in the center area, as previously described. ${ }^{27}$ Additionally, $\mu \mathrm{IM}$ specimens of the same samples were fine-polished with 600 mesh sandpaper to remove a layer of 50-60 $\mu \mathrm{m}$ thickness on each side of the $1 \mathrm{~mm}$ wide specimen surface, referred to as ' $\mu \mathrm{IM}$ core,' were also analyzed. Extruded samples, in the form of a continuous rod with approximately $500 \mu \mathrm{m}$ thickness, of the same nanocomposites above were cut perpendicularly to the extrusion direction and fixed in the beam path using tape. Background scattering was acquired and subtracted from the samples scattering patterns. The sample plane was fixed perpendicular to the X-ray beam and positioned at $70 \mathrm{~mm}$ from the CCD detector. The diffraction patterns were acquired using 30 and $10 \mathrm{~s}$ collection times for microinjected and extruded samples, respectively.

From the XRD data, quantitative evaluations of the two crystalline forms content were obtained by curve fitting of the integrated intensity profiles. Two Gaussian functions were used for the $\alpha$ phase, two for the $\gamma$ phase, and one for the amorphous halo, and fitted against the integrated intensity profile to calculate the area of each peak.

Orientation was calculated at the $\gamma_{2}, \alpha_{1}$, and $\alpha_{2}$ peaks position based on the azimuthal distribution of intensity using POLAR software. ${ }^{32}$ 


\section{Results and discussion}

Dispersion of carbon nanotubes in polyamide 6

The dispersion of $\mathrm{p}$-CNT and f-CNT in PA6 was quantitatively assessed in a previous work ${ }^{29}$ that revealed an influence of CNT functionalization, and an even more pronounced effect of the processing method. Hence, the $\mu \mathrm{IM}$ step performed after composite extrusion improved the level of CNT dispersion, with a stronger effect on $\mathrm{f}$-CNT. This effect was not systematically observed for the extruded composites. The expected role of the cyclic amine bonded to the CNT surface was to increase the CNT/polymer interfacial strength through hydrogen bonding. Covalent bonding of the cyclic amine and carboxylic endgroups of the PA6 may also take place under melt processing conditions. SEM images of cryo-fractured nanocomposite surfaces, presented in Fig. 1, illustrate the PA6/p-CNT and PA6/fCNT interface. The white spots correspond to nanotube ends that were pulled out of the PA6 matrix. f-CNT appear to be better wet by the PA6 matrix compared to $\mathrm{p}-\mathrm{CNT}$, considering the shorter CNT pull-out length, regardless composition.

\section{Differential scanning calorimetry}

The influence of $p$-CNT and $f-C N T$ on the crystallization and melting behavior of PA6 processed by extrusion and by $\mu \mathrm{IM}$ at two different CNT concentrations was investigated by DSC. Figure 2 shows the DSC curves of pure PA6 and PA6/CNT nanocomposites during heating above the melting temperature to study the effect of thermal history induced during processing on the extruded and $\mu \mathrm{IM}$ nanocomposites.

The temperature of highest heat flow $\left(T_{\mathrm{m}}\right)$ and the enthalpy of fusion $\left(\Delta H_{\mathrm{f}}\right)$, during melting, and the crystallization temperature $\left(T_{\mathrm{c}}\right)$ and the enthalpy of crystallization $\left(\Delta H_{c}\right)$, during cooling, were measured. The $\chi_{c}$ was calculated from the enthalpy of fusion $\left(\Delta H_{f}\right)$ according to equation (1). The results obtained are presented in Table 1.

The melting temperature, determined from the melting peak is similar for PA6 and PA6/CNT nanocomposites for both processing techniques, and all materials start melting

P1
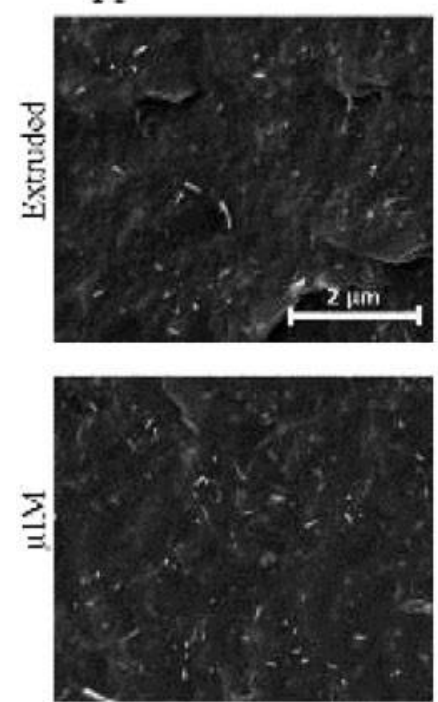

$\mathrm{F} 1$
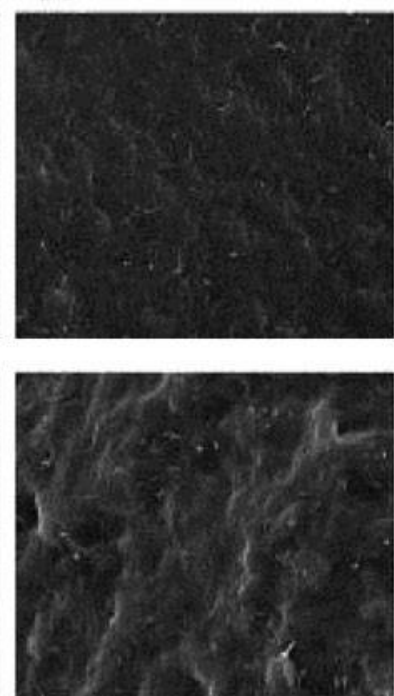

near $203 \complement$. The major difference is that $\mu$ IM PA6 presents a small secondary crystallization peak starting near $190 \mathrm{X}$. This secondary crystallization was observed as well for the $\mu \mathrm{IM}$ composites, particularly with low CNT content. Secondary crystallization may be associated to the presence of microcrystals formed under the $\mu \mathrm{IM}$ shear stresses, which crystallize during sample heating. ${ }^{33}$ It may also be associated to the conversion from $\gamma$ to $\alpha$ phase during heating, as reported by Xie et al. $^{34}$ The heat of fusion and corresponding crystallinity reported in Table 1 for PA6 and the composites with $1 \mathrm{wt}-\%$ of CNT were corrected for the occurrence of the secondary crystallization. This effect is considerably smaller for the samples with high CNT content, for which this correction could not be applied. The DSC measurements of the $\mu \mathrm{IM}$ samples are affected by this secondary crystallization, making it difficult to correctly quantify the overall crystallinity of these samples. More accurate crystallinity measurements will be obtained by WAXD, as they are performed without perturbation of the polymer crystalline structure.

The effect of $\mu \mathrm{IM}$ on the degree of crystallinity, as measured by DSC, is shown in Table 1. It was observed that the $\mu \mathrm{IM}$ process does not considerably affect the overall degree of crystallinity of the nanocomposites, in agreement with other results reported in the literature. ${ }^{22,23}$ The effect of CNT addition slightly increased the overall degree of crystallinity measured by DSC, but the effect was not substantial. ${ }^{22}$

The effect of nanotube loading on the crystallization of PA6/CNT nanocomposites was examined by DSC after eliminating the thermal history, i.e. after melting and holding the temperature for $2 \mathrm{~min}$. Figure 2 shows the DSC thermograms recorded during controlled cooling of extruded and $\mu \mathrm{IM}$ samples of $\mathrm{p}$-CNT and $\mathrm{f}-\mathrm{CNT}$ at two nanotube loadings. It was observed that cooling of PA6 under quiescent conditions originates a single crystallization peak with maximum intensity near $193 \mathcal{C}$, while PA6/CNT nanocomposites display a crystallization process that starts at a higher temperature, approximately $209 \mathrm{C}$, and has a second and more intense peak near $197 \mathfrak{C}$. A similar behavior is

P4.5
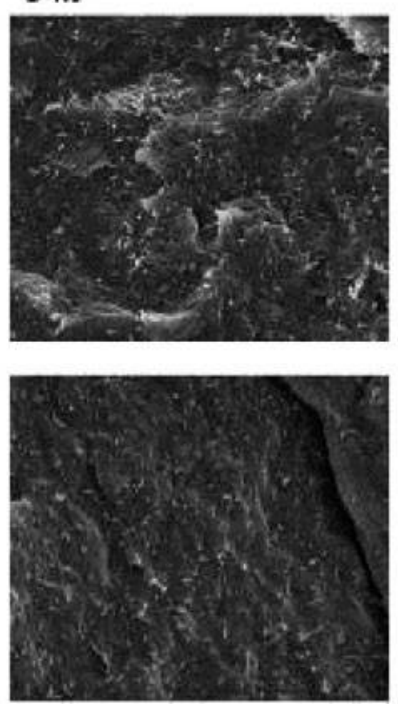

F 4.5
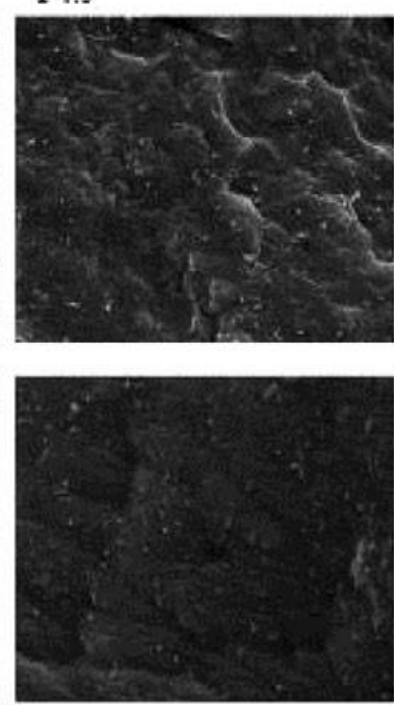

Figure 1 SEM micrographs of the composites with 1 and $4.5 \mathrm{wt}-\%$ of pristine and functionalised carbon nanotube (p-CNT and f-CNT) (designated as P1, F1, P4.5, and F4.5) obtained for extruded and microinjection molding ( $\mu$ IM) samples. All the SEM images have the same scale as the first 

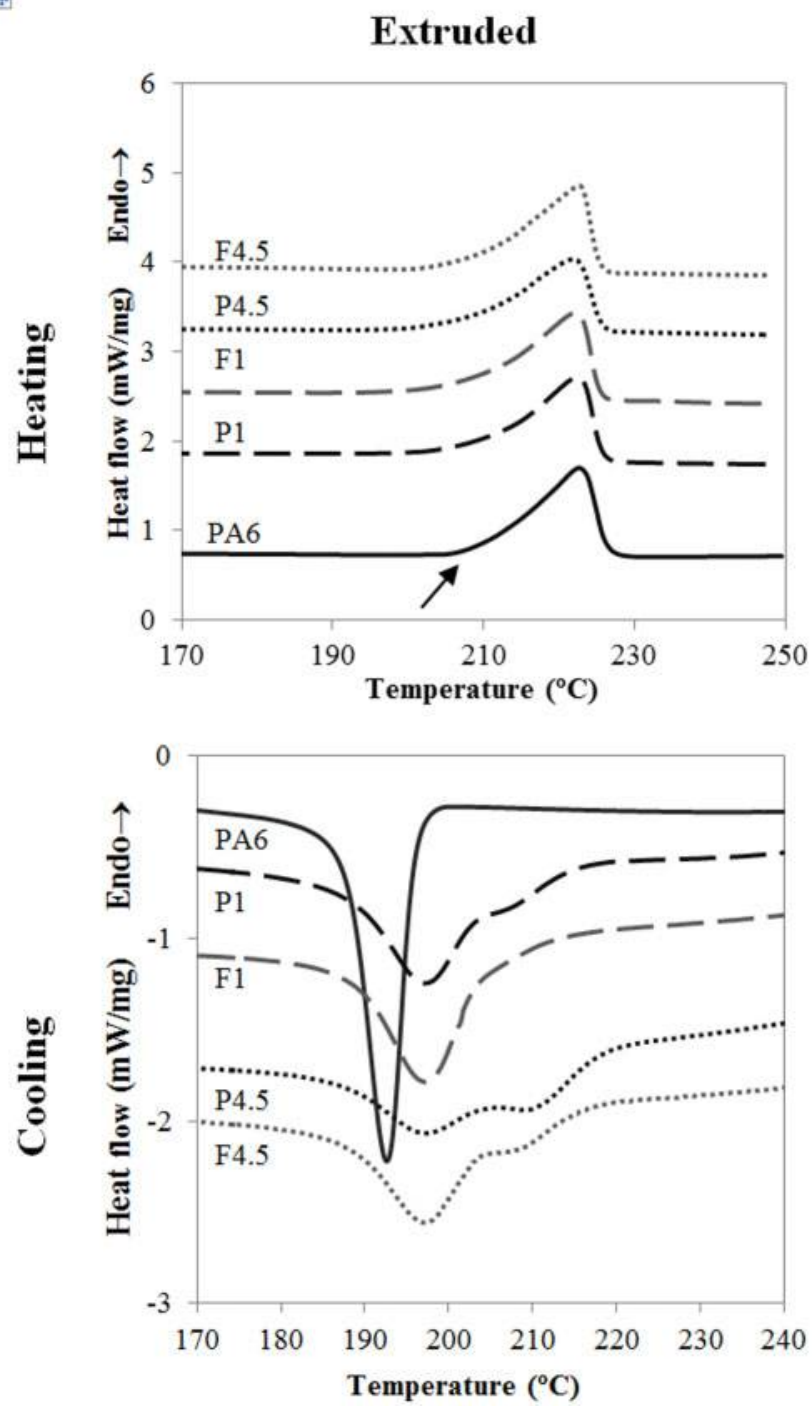

$\boldsymbol{\mu}$ Injection Moulded
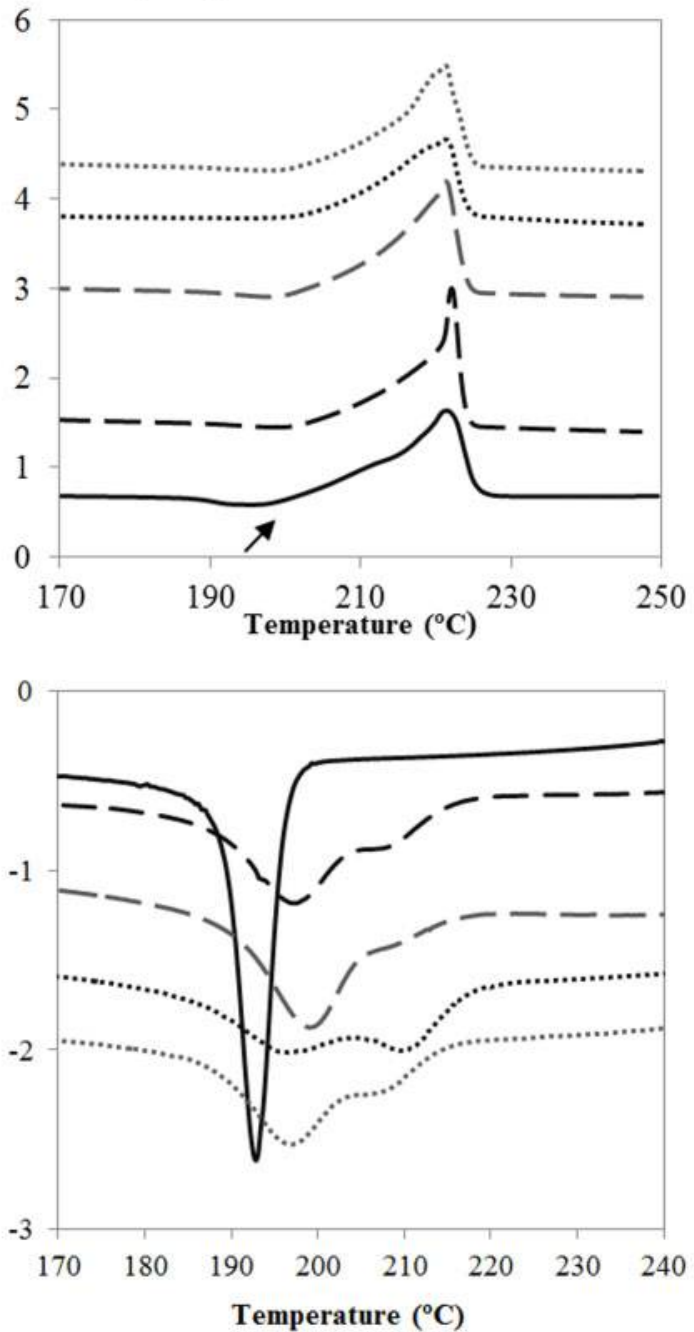

Figure 2 Differential scanning calorimetry (DSC) thermograms of PA6 and PA6 with pristine and functionalised carbon nanotube ( $p$-CNT and $f-C N T)$ for extruded and microinjection molding $(\mu \mathrm{IM})$ nanocomposites, under controlled heating and cooling from the melt

Table 1 Melting and crystallization characteristics of the extruded and microinjection molding $(\mu \mathrm{IM})$ PA6 and nanocomposites

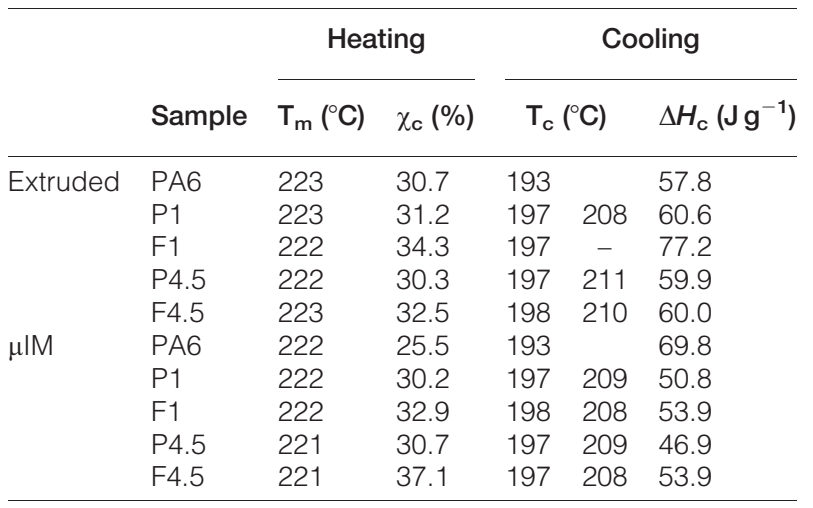

observed either for extruded or $\mu \mathrm{IM}$ composite samples. These observations demonstrate the homogeneity of the crystallization process in pure PA6 (under quiescent conditions) and the role of the large graphite-like surface of the CNT that assist the crystallization process, inducing it at higher temperature. This effect is more evident for the higher CNT content composites. Table 1 show that the incorporation of CNT results in a rise of $T_{\mathrm{c}}$ for all nanocomposites, and that this increment is similar for all nanocomposites, extruded or $\mu \mathrm{IM}$. PA6 crystallization temperature interval is wider for all nanocomposites than for PA6 alone. This behavior of PA6 in the presence of CNT was reported before, ${ }^{10,12,13}$ and was interpreted in two different ways. The CNT surface may induce crystallite growth perpendicular to the CNT axis leading to the development of transcrystallinity, ${ }^{12,35}$ while away from the CNT crystal growth occurs in the bulk polymer. Another interpretation, by Phang et $a l_{1}^{10}$ explains the double crystallization peak simply as a two-step crystallization of $\alpha$ phase.

\section{Wide angle X-ray diffraction}

WAXD patterns and the corresponding integrated intensity profiles obtained for the extruded and $\mu \mathrm{IM}$ samples are presented in Fig. 3. The integrated intensity profiles demonstrate that the $\gamma$ crystalline form predominates in the $\mu \mathrm{P}$ of PA6, while the contribution of the $\alpha$ form dominates in the $\mu \mathrm{IM}$ 

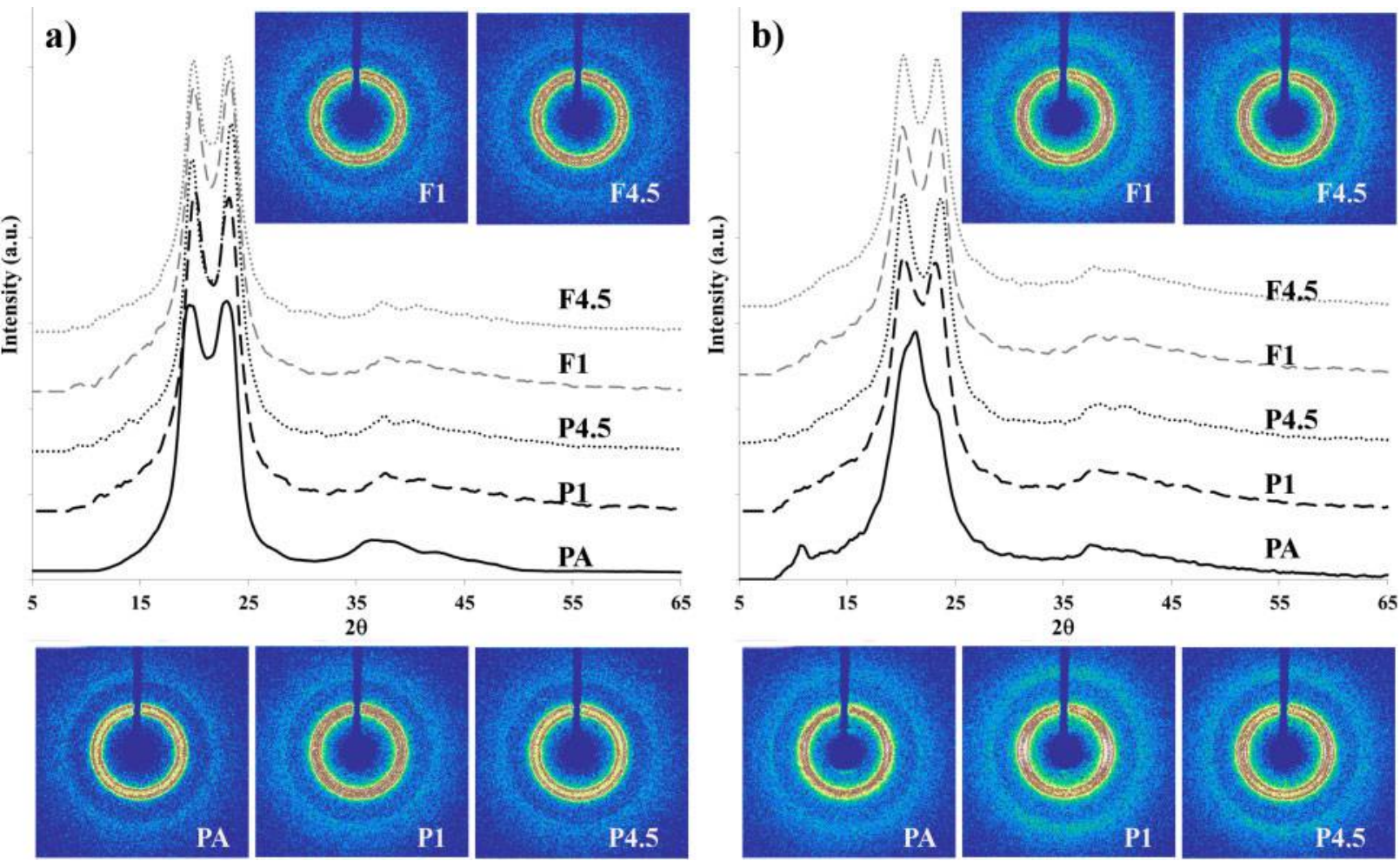

Figure 3 Wide angle X-ray diffraction (WAXD) patterns and integrated intensity profiles of extruded a and microinjection molded $b$ samples

nanocomposites, and all extruded samples. Orientation effects are observed for all $\mu \mathrm{IM}$ samples.

The orientation of the crystalline reflections was determined using POLAR software performing the analysis on the $2 \mathrm{D}$ intensity data. The measured intensities were decomposed in two components, one isotropic and another anisotropic. The Hermans orientation factors, $f_{\mathrm{z}}{ }^{36}$ calculated on the anisotropic intensity component, are presented in Table 2 for the $\mu \mathrm{P}(\mu \mathrm{IM})$, its core region ( $\mu \mathrm{IM}$ core), and for the extruded sample (extrusion) for all compositions.

All $\mu \mathrm{IM}$ samples present considerable orientation, higher for the data collected on the overall sample thickness compared to the data from the core region alone. The extruded samples do not show significant orientation. Orientation slightly decreases with CNT content and functionalization. Comparing the orientation factors obtained for the overall sample and the core, the results evidence a larger difference between these values for PA6 compared to the high and low loading nanocomposites. For the high-loading composites, no appreciable difference is measured for core and overall sample orientation. The orientation effect for the $\mu \mathrm{IM}$ samples at the core region increases when CNTs are present. This effect may relate to CNT orientation along the flow during $\mu \mathrm{IM}$, inducing oriented crystalline growth upon cooling.

The crystallinity calculated from the WAXD results for the nanocomposite samples is lower compared to pure PA6 sample, regardless of the processing technique or the region of measurement (Fig. 4). The results for the $\mu \mathrm{IM}$ samples also show a marked difference for the proportion of $\gamma$ and $\alpha$ phases, the $\gamma$ phase is dominating in the pure PA6 sample, while for all composites, the $\alpha$ phase is more abundant. The $\gamma$ phase content is similar for all the composites samples except the overall $\mu \mathrm{IM}$ composite with $1 \mathrm{wt}-\% \mathrm{p}-\mathrm{CNT}$ that displays higher $\gamma$-phase content, as can be observed in Fig. 4. The extruded material shows in general lower $\gamma$ phase content along with higher total crystallinity compared to the $\mu \mathrm{IM}$ samples, and thus a larger $\alpha$-phase to $\gamma$-phase ratio. Carbon nanotubes functionalization favors a slightly higher overall crystallinity for both the extruded and $\mu \mathrm{IM}$ nanocomposites.

Table 2 Hermans orientation factors* calculated for microparts $(\mu \mathrm{P})$ and extruded PA6 samples

\begin{tabular}{|c|c|c|c|c|c|c|}
\hline \multirow{3}{*}{$\begin{array}{l}\text { Samples } \\
\text { PA6 } \\
\text { P1 }\end{array}$} & \multicolumn{2}{|c|}{ Microinjection molding $(\mu \mathrm{IM})$} & \multicolumn{2}{|c|}{$\mu \mathrm{IM}$ core } & \multicolumn{2}{|c|}{ Extrusion } \\
\hline & $-0.2021(\gamma 2)$ & & $-0.1165(\gamma 2)$ & & -0.0695 & 0.0723 \\
\hline & -0.2425 & -0.2794 & -0.1854 & -0.2288 & 0.1329 & -0.095 \\
\hline P45 & -0.2183 & -0.2171 & -0.1799 & -0.1648 & 0.0196 & 0.0112 \\
\hline $\mathrm{F} 1$ & -0.2081 & -0.2047 & -0.1990 & -0.1829 & -0.0455 & -0.0570 \\
\hline F45 & -0.1815 & -0.1651 & -0.1643 & -0.1958 & -0.0162 & 0.1258 \\
\hline
\end{tabular}

* The Hermans orientation factor ranges from -0.5 to 1.0 , corresponding to fully oriented crystalline planes perpendicular and parallel to the reference axis, respectively. A value of zero corresponds to random orientation. Except otherwise indicated, the orientations were calculated at the $\alpha_{1}$ and $\alpha_{2}$ peaks, $2 \theta=20.25^{\circ}$ and $23.26^{\circ}$, respectively. The values marked as $\gamma_{2}$ were calculated using the $\gamma_{2}$ peak at $2 \theta=21.25^{\circ}$. 


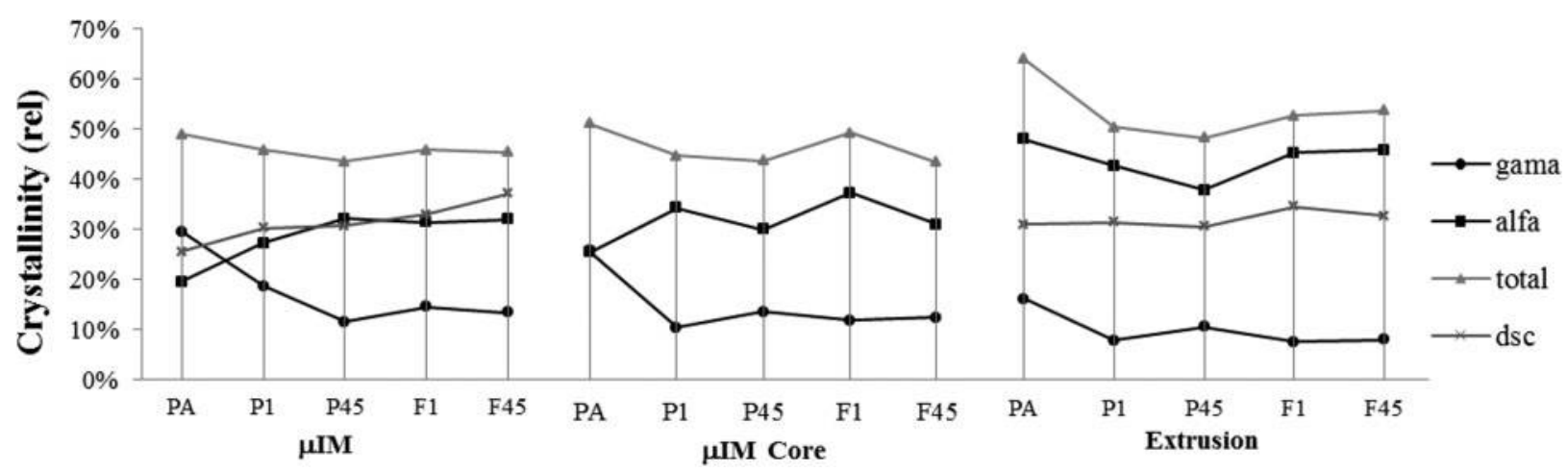

Figure 4 Overall crystallinity of $\alpha$ - and $\gamma$-phase content data obtained from wide angle X-ray diffraction analysis (WAXD) results

The overall higher crystallinity observed for the extruded compared to $\mu \mathrm{IM}$ composites and PA6 may be associated to lower cooling rate induced by the former process, thus allowing longer crystallization time. The lower $\gamma$-phase content observed for the extruded materials strengthens this hypothesis. While the extruded polymer and composite rod has a diameter larger than $2 \mathrm{~mm}$, the $\mu \mathrm{P}$ thickness is $300 \mu \mathrm{m}$, and cooling occurs in close contact with the cooled and highly thermally conductive mold wall.

Regardless of the processing method, the crystallinity measured by WAXD is lower for the nanocomposites compared to pure PA6. This effect may be associated to the high thermal conductivity of the CNT that may reduce the cooling time of the nanocomposites.

\section{Conclusions}

Differential scanning calorimetry and wide angle X-ray diffraction were successfully used to analyze the morphology induced by the processing method in PA6 and PA6/CNT composites for extrusion and microinjection molding.

Differential scanning calorimetry analysis showed that $\mu \mathrm{IM}$ samples with low CNT content presented a secondary crystallization process at a temperature just below the onset of the melting peak, which was not observed for the extruded materials. Controlled cooling of the PA6 and composite melts revealed the important differences in the crystallization process in the presence of CNT, which induced crystallization at higher temperature.

The overall crystallinity measured by wide angle X-ray diffraction was considerably higher for the extruded materials compared to the corresponding $\mu \mathrm{IP}$, and the main contribution to this difference was the larger amount of $\alpha$-phase crystallinity. The $\gamma$-phase contribution was similar for the materials obtained by both the processing methods (slightly smaller for the extruded material).

The major morphological dissimilarity between extruded and $\mu \mathrm{IM}$ materials was observed for PA6 without CNT. The $\mu \mathrm{M}$ PA6 developed a large fraction of $\gamma$-crystalline phase, mostly at the sample skin region. This effect was not observed for the nanocomposites, either with $\mathrm{p}$-CNT or $\mathrm{f}-\mathrm{CNT}$.

Functionalization of the CNT did not affect the polymer morphology, inducing the formation of a marginally larger amount of $\alpha$ phase compared to $\mathrm{p}-\mathrm{CNT}$.

\section{Conflict of interest}

The authors declare that they have no conflict of interest.

\section{Acknowledgments}

The authors acknowledge Fundação para a Ciência e Tecnologia for project PEst-C/CTM/LA0025/2013 (Strategic Project - LA 25 - 2013-2014), and for PhD grant for T. Ferreira (SFRH/BD/39119/2007). We acknowledge University of Aveiro, Department of Chemistry/CICECO for the use of the X-ray equipment, in particular Dr Paula Brandão, for helping on the X-ray measurements.

\section{References}

1. M. Paradise and T. Goswami: Mater. Des., 2007, 28, 1477-1489.

2. R. H. Baughman, A. Zakhidov and W. A. Heer: Science, 2002, 297, 787-792.

3. M. Endo, M. S. Strano and P. Ajayan: 'Potential applications of carbon nanotubes', in 'Carbon Nanotubes (111, Topics in Applied Physics)', (eds. A. Jorio, G. Dresselhaus and M. S. Dresselhaus), 13-62; 2008, Berlin, Springer-Verlag.

4. P. Chen, H. S. Kim and H. J. Jin: Macromol. Res., 2009, 17, 207-217.

5. J. Giboz, T. Copponnex and P. Mélé: J. Micromech. Microeng., 2007, 17, R96-R109.

6. G. Trotta, R. Surace, F. Modica, R. Spina and I. Fassi: 'Micro injection moulding of polymeric component'. Proceedings of International Conference on Advances in Materials and Processing Technologies (AMPT2010), Paris, France, AIP Conference Proceedings, 2010, 1315, 1273-1275.

7. A. J. Pontes, A. T. Sepúlveda, M. Sampaio, J. Cortez and A. M. Cunha: 'Analysis of morphology and performance of micro dumbbell specimens manufactured by micro injection molding', Proceedings of the 24th Annual Meeting of the Polymer Processing Society, Salerno, June 2008 15-19.

8. M. Ito, K. Mizuochi and T. Kanamoto: Polymer, 1998, 39, 4593-4598.

9. N. S. Murthy: J. Polym. Sci. B Polym. Phys., 2006, 44, 1763-1782.

10. I. Y. Phang, J. Ma, L. Shen, T. Liu and W. Zhang: Polym. Int., 2006, 55, 71-79.

11. H. Ha and S. C. Kim: Macromol. Res., 2010, 18, 660-667.

12. E. Logakis, C. Pandis, V. Peoglos, P. Pissis, C. Stergiou, J. Pionteck, P. Pötschke, M. Micusík and M. Omastová: J. Polym. Sci. B Polym. Phys., 2009, 47, 764-774.

13. X. Q. Liu, W. Yang, B. H. Xie and M. B. Yang: Mater. Des., 2012, 34, 355-362.

14. D. M. Lincoln, R. A. Vaia, Z. G. Wang and B. S. Hsiao: Polymer, 2001, 42, 1621-1631.

15. T. D. Fornes and D. R. Paul: Polymer, 2003, 44, 3945-3961.

16. T. Liu, I. Y. Phang, L. Shen, S. Y. Chow and W. D. Zhang: Macromolecules, 2004, 37, 7214-7222.

17. J. N. Coleman, M. Cadek, K. P. Ryan, A. Fonseca, J. B. Nagy, W. J. Blau and M. S. Ferreira: Polymer, 2006, 47, 8556-8561.

18. N. Roya, R. Senguptaa and A. K. Bhowmick: Prog. Polym. Sci., 2012, 37, 781-819.

19. P. C. Ma, N. A. Siddiqui, G. Marom and J. K. Kim: Compos. Part A, 2010, 41, 1345-1367. 
20. J. Li, Z. Fang, L. Tong, A. Gu and F. Liu: J. Polym. Sci. B Polym. Phys., 2006, 44, 1499-1512.

21. J. Li, C. Ke, K. Fang, X. Fan, Z. Guo and Z. Fang: J. Macromol. Sci. Phys. 2010, 49, 405-418.

22. H. Meng, G. X. Sui, P. F. Fang and R. Yang: Polymer, 2008, 49, 610-620.

23. H. Liu, X. Wang, P. Fang, S. Wang, X. Qi, C. Pan, G. Xie and K. M. Liew: Carbon, 2010, 48, 721-729.

24. F. Liu, C. Guo, X. Wu, X. Qian, H. Liu and J. Zhang: Polym. Adv. Technol., 2012, 23, 686-694.

25. J. Zhang, L. Zhang, H. Liu, F. Liu and C. Guo: Polym. Plast. Technol. Eng., 2012, 51, 1032-1037.

26. Z. Lu and K. F. Zhang: Polym. Eng. Sci., 2009, 49, 1661-1665.

27. T. Ferreira, P. E. Lopes, A. J. Pontes and M. C. Paiva: Polym. Adv. Technol., 2014, 25, 891-895.

28. S. Abbasi, A. Derdouri and P. J. Carreau: Polym. Eng. Sci., 2011, 51, 992-1003.
29. T. Ferreira, M. C. Paiva and A. Pontes: J. Polym. Res., 2013, 20, 301-310.

30. M. Paiva, F. Simon, R. Novais, T. Ferreira, M. Proença, W. Xu and F. Besenbacher: ACS Nano, 2010, 4, 7379-7386.

31. P. G. Galanty: 'Nylon 6' in 'Polymer data handbook', (ed. J. E. Mark), 181; 1999, Oxford University Press, New York, NY, USA.

32. S. Raj: 'X-ray data analysis software manual'; 2003, Stony Brook, NY, USA, Stonybrook Technology and Applied Research, Inc. v 1.04.

33. S. T. Correale and N. S. Murthy: J. Appl. Polym. Sci., 2006, 101, 447-454.

34. S. Xie, R. Séguéla, J. M. Lefebvre and J. M. Gloaguen: J. Polym. Sci. B Polym. Phys., 2009, 47, 2385-2393.

35. S. Zhang, L. M. Minus, L. Zhu, C. P. Wong and S. Kumar: Polymer, 2008, 49, 1356-1364.

36. L. E. Alexander: 'X ray diffraction methods' in polymer science'; 1969, New York, Wiley-Interscience. 